\title{
Constituting Factors of a Digitally Influenced Relationship between Patients and Primary Care Physicians in Rural Areas
}

\author{
Marius Mueller, Michael Knop, Caroline Reßing, Henrik Freude, Frederike Marie Oschinsky, \\ Hans Christian Klein, Bjoern Niehaves \\ University of Siegen \\ \{marius.mueller | michael.knop | caroline.ressing | henrik.freude | frederike.oschinsky | \\ christian.klein | bjoern.niehaves\} @ uni-siegen.de
}

\begin{abstract}
In times of an ageing society and a rural exodus of primary care physicians, healthcare systems are facing major challenges. To maintain comprehensive care and an equitable access to healthcare services, today's technological advancements represent a promising measure. Technologies empower patients by providing innovative tools such as sensors and applications for self-measurement, leading to selfinitiated interventions, while supporting physicians in handling rising demands through telemedicine and spatially detached solutions. These enhanced treatments come with patient and physician-sided challenges such as incorrect digital information provided to the patient, negatively affecting treatment quality and leading to high issue resolving efforts. In order to investigate the perspectives of rural physicians on treatment digitalization and effects of patient empowerment, we conducted a qualitative study using semi-structured interviews. Our findings show that patient activation, impacts on treatment process, patient differentiation, and patient-physicianinteraction are relevant factors in the physicians' valuation and willingness to use health technologies.
\end{abstract}

\section{Introduction}

In times where digitalization and innovative tools have an impact on multiple areas of life, technological advancements promise benefits for therapeutic treatment processes and healthcare in general. The possibility to overcome long distances, while technical limitations are declining due to structural expansions and governmental investments, enable an unprecedented and timely alternative to common care structures and processes. Today's healthcare systems exhibit shortcomings that threaten the maintenance and stable provision of comprehensive care. In many countries and rural regions in particular, the endemic healthcare system is hardly suited to adequately face ongoing demographic changes and the rising numbers of age-related health issues [9]. Increasing patient numbers lead to a demand surpassing the current healthcare supply, which is reinforced by medical professionals and graduates moving to urban and often more attractive regions. The resulting rural undersupply leads to a spatially as well as temporally limited access to care and an inequitable distribution of care facilities [36]. This development increasingly pressures practicing physicians and professionals.

Technologies potentially provide alternatives to analogous, location-based, and often unilateral care [20]. For instance, modern communication tools such as live video conferencing enable geographically detached physician consultations [18]. Body-worn sensory equipment, ranging from medical products developed for health and treatment purposes to consumer technologies such as smartwatches [27], further enable mutual therapeutic processes in which patients take more active roles by measuring their personal vital signs [23], informing themselves about health issues and treatments [1], and showing increasing degrees of overall health literacy [17]. Both examples show how to overcome mobility issues caused by illness or infrastructural shortages.

Since digital innovations empower patients and physicians in either managing their condition or performing treatments and work processes in the face of high demand, new questions and challenges arise. On the consumer side, patients are confronted with a plethora of available technologies and health-related information offered by the internet and mHealth applications. Difficulties arise when a user needs to judge on what technologies to use and how, which represents a major factor in ageing societies [14]. Furthermore, while potentially delivering benefits for effective self-management and behavior in case of health issues, the assessment of information and data can lead to misinterpretation, misguidance, or excessive demand, while information quality and correctness is oftentimes questionable [29]. On the 
provider side, physicians and medical staff are obliged to incorporate technological solutions into their work routines, supplementing or even altering their way of working. They need to acquire competences to implement digital processes and to use the provided devices in an efficient and appropriate way [6], while guidance and financial support is oftentimes scarce [31]. In addition, physicians have to deal with issues arising from patient-sided activities and empowerment. Problems originating in misusing, misinterpreting, or relying on flawed information lead to an increased workload to resolve these issues and safeguard treatment quality and patient health [1].

Consequently, bringing together challenges in rural areas and the perceptions of physicians regarding the application of health technologies for patient treatment as two major factors influencing successful IT implementation and adoption, the physician perspective needs to be illuminated in more detail. To date, research on how physicians in rural areas perceive, anticipate, and evaluate treatment digitalization and accompanying patient empowerment under the light of emerging challenges is scarce. We conducted an explorative, qualitative study in the form of seven semi-structured interviews to investigate and fill this gap. Thus, our paper contributes to our understanding of the potential impacts, benefits, and issues that arise from digital interventions in rural areas, enabling the design of needs-based and acceptable solutions in times where IT adoption rates in healthcare are low [16]. To gather perspectives rural physicians have on applying technologies in patient treatment, the interview guideline used in our study covers questions on (1) the interviewees' general perceptions regarding rural healthcare, digitalization, job and patient related factors, and (2) assessments of a concrete technical setting. This setting describes a video-conferencing tool that can be used for patient-physician communication, supplemented by sensors that capture data and transfer them to the physician. We see our paper as an initial step of investigation where we focus on the physician side in particular. Perspectives of the patients, thus, remain a future research topic enabling comparative studies and insights. The study at hand is guided by the following research question (RQ):

RQ: What factors constitute the perspectives of physicians on the utilization of patient-empowering digital technologies in rural areas?

\section{Theoretical Background}

As a theoretical baseline for our study, we look at related work concerning patient empowering effects of healthcare and treatment digitalization as well as specifics of rural areas that affect the applicability, necessity, and evaluation of digital interventions.

\subsection{Digital Patient Empowerment}

Whereas traditional treatment settings commonly involve bilateral relationships between patients and physicians based on interpersonal factors [13], the introduction of digital technologies into therapeutic processes creates trilateral scenarios by introducing health technologies as a third actor in the treatment process [34]. Besides the physician and the patient, technologies can take active (e.g., by actively informing the user when certain parameters surpass thresholds) and/or passive roles (e.g., by solely reacting to user queries such as health information retrieval) supplementing treatments by, for instance, measuring health data $[22,35]$, delivering information [19], or enabling communication [18].

Health technologies and digitized treatment support enable a deliberation of healthcare service provision as well as treatment execution and adjustment, empowering patients to take more care of themselves and increase their levels of health efficacy. As a result, "[a]dvances in technology have empowered patients to be informed, which enabled them to play an active role in clinical encounters with the doctor" (p. 1) [26]. As an essential characteristic of the deliberative physician-patient relationship model, Emanuel and Emanuel [10] describe, that "[...] the aim of the physician-patient interaction is to help the patient determine and choose the best healthrelated values that can be realized in the clinical situation" (p. 2222). The model stipulates that the physician should suggest which health-related values should be pursued and, based on that, figure out the best and most desired way of treatment in cooperation with the patient. Physicians and patients step into a cocreation of therapeutic treatment [26].

Looking at current advancements in digitally supplemented healthcare such as the technologyenabled self-measurement of vital parameters (e.g., via smartwatch) or looking up and discussing healthrelated information online, deliberation takes place in a new form. Values with regard to diseases, appropriate therapeutic measures, and desired outcomes can be increasingly generated and assessed by the empowered patient [34]. Through activities like information seeking and accessing health-related information via the internet or peers, patients oftentimes form expectations and preconceptions on (a) their condition and (b) what treatment allegedly suits them best before even consulting a physician or therapist [34]. As a result, patient empowerment leads 
to an increase in patient-sided sense of mastery and control as well as self-efficacy and potentially improved decision-making [5]. This development has several implications for both healthcare providers and consumers. While digital tools have the potential to improve and, in case of rural undersupply and scarce access to care, enable treatments without temporal and spatial bounds, technologies can have negative impacts as well. Misunderstandings and potentially harmful actions patients may take based on flawed information [29] have to be counteracted by physicians and technology providers as well [1]. Consequently, the examination of patient and physician perspectives on treatment digitalization is of major importance for health technology design, application, and evaluation.

\subsection{Specifics of Rural Healthcare}

Rural areas exhibit several characteristics that influence the applicability and necessity of digital interventions as a supplement for care processes and structures. In addition, the given rural circumstances potentially shape the way people perceive, adopt, and use technologies implemented to support treatments and enable access to care.

Healthcare issues in rural areas appear in different forms. For instance, environmental, geographical, and infrastructural circumstances can lead to adverse and inequitable spatial distributions of care facilities and professionals [36]. Patients as well as physicians are obliged to travel long distances either to consult a professional or to visit patients in need of care at home $[3,7]$. Furthermore, many physicians and young medical graduates tend to practice in urban or suburban areas [30]. The rural exodus of healthcare professionals, inter alia, is driven by a huge (on-call) demand for care while supply is scarce, leading to exhaustion and work-life-balance issues [33], and (perceived) benefits of structurally stronger regions such as the quality of education [37], attracting physicians to settle. In addition, rural areas suffer from inferior access to specialized and appropriately educated healthcare providers as opposed to urban areas [15]. Hence, the rural population faces greater issues regarding the availability of specialized practitioners (e.g., cardiologists), leading to a lack of supply beyond treatment of common diseases and basic care. Further, studies report on barriers towards healthcare that rural areas struggle more with compared to urban areas. Besides others, resource limitations (e.g., the lack of colleagues that physicians can consult for council), confidentiality limitations (i.e., concerns about reporting sensible data to authorities), and overlapping roles (i.e., physicians meeting clients in private life contexts) are increasingly noticed [3]. As a result, disparities with regard to access to care and population-wide health status emerge [28] and "[...] traditional concerns about access to primary and hospital care continue to dominate rural health policy" [12] (p. 1675).

However, while potentially delivering benefits for maintaining a comprehensive care in the near future, technologically supported treatments come with challenges. For instance, digital tools require a certain degree of skill and efficacy, rendering a proportion of patients and physicians unable to use them. Especially in times of ageing societies, which particularly emerge in many rural areas across both developed and developing countries [2, 8, 39], this issue becomes apparent and calls for higher involvement and guidance $[14,25]$. In addition, valuation of technology is often rooted in social cues and opinions that affect users' adoption and use behavior. This effect is potentially reinforced by rural structures, where health literacy can be low [38] and trust often solely roots in statements and assessments by professionals [13]. This can impede the effectiveness of health information delivered by technologies. However, as studies have shown, the willingness and confidence to use telemedical systems does not significantly differ when comparing rural and urban populations [11], showing that the path for telemedical systems in healthcare can be made once sufficient education is provided and awareness granted [21].

The circumstances found in rural areas and populations described above illustrate the need for a dedicated investigation of rural areas as a reasonable space for digitalization. Literature has unveiled significant issues and barriers healthcare providers as well as consumers have to deal with, further motivating the study at hand. Apparently, physicians are facing major challenges when providing comprehensive care, reinforcing the issues associated with the future application of health technologies for patient treatment. Their perspectives on the potentials as well as constraints of health technologies are identified as a major scientific and practical demand.

\section{Methods}

\subsection{Case Description}

We conducted a qualitative study involving seven semi-structured interviews with primary care physicians. The study took place within a regional project on digitalization of primary care practices and processes. One major focus is the investigation of health technology acceptance by rural primary care 
physicians, which are potentially obliged to implement, adopt, and continuously use technological tools in the near future due to declining amounts of accessible physicians. Here, the perceived influence and impact of digital tools on the patient-physician relationship, the treatment process, and the physicians' performance form promising predictors of technology acceptance and adoption behavior.

The technological setting that our empirical investigation is based on consists of three incremental versions of a telemedicine system for patient communication, treatment, and diagnosis: (1) Establishing a live video conference between a spatially separated physician and patient, enabling face-to-face communication and basic examinations (e.g., check for wounds or skin abnormalities via webcam). (2) Enhancing the video solution with bodyworn sensors that are able to measure relevant vital parameters (e.g., blood pressure). The captured data can be transmitted to the physician without temporal restrictions, enabling an immediate inspection by the physician. As a result, the physician can adjust the treatment and, if needed, arrange a physical meeting. (3) Analyzing the data right after its acquisition, for instance by using Artificial Intelligence (AI) algorithms that pre-analyze the data before the results are transferred. Consequently, the physician has the option to only receive information gathered from the raw data, such as incidents where given vital parameters surpass critical thresholds and call for intervention. This contains the potential to save valuable time since the expert does not have to look through all the data arriving throughout the day.

\subsection{Data Collection and Analysis}

The seven interviews took between 50 and 120 minutes (75 minutes on average) and were conducted on the physician site. We followed a convenient sampling approach. Interviewees were recruited via a regional governmental gatekeeper reaching out to physicians willing to participate in the aforementioned project. The sample yielded three female and four male interviewees. Interviewees were aged between 41 and 66 years ( 52 years on average) and had between 15 and 34 years (25 years on average) of job experience as a physician. Due to the recruitment process, all participants were located in the same region dealing with comparable circumstances. As a prerequisite to gather meaningful and rich data, all participants exhibit a sufficient degree of technical affinity and interest enabling them to fathom the technological setting and potential impacts.

The participants were interviewed in two blocks on two consecutive days due to temporal limitations originating in the physicians' time schedule and obligations. The interview guideline was not changed between sessions, representing a non-iterative procedure. This led to an ex post data analysis that was performed after the last interview had been conducted. In doing so, we tried to increase variance in physician perceptions while reducing bias by extending interviews with previous findings.

The interview process consisted of two phases. In phase one, the interviewees were asked about their general opinion on the state of rural healthcare, associated opportunities and challenges for change, and their own job related conditions. In addition, we asked for the participants' view on digitalization, particularly in healthcare and patient treatment. Sample questions are "What chances and risks do you see with regard to progressing digitalization?" or "Are you willing to digitalize your practice in the future?" In phase two, questions revolved around the technical setting described above. After introducing the scenario involving three incremental system versions, interviewees were asked to assess the described digitized setting with regard to feasibility, functionality, usefulness, as well as arising challenges and issues that are associated with implementing and using the system. Sample questions are "Do you consider the described system suitable for daily use?" or "What do you think is important for patients to accept the system?" This two-phased approach allowed us to gradually increase the questions' reference to digitalization, thus gathering more general as well as specific data on the physicians' perspectives on health technology. The interviews were conducted in German, audio recorded, and transcribed nonverbatim while containing the meaning and formulation. For the purpose of analysis, the data was translated into English.

For data analysis, we followed a Grounded Theory approach consisting of open, axial, and selective coding [32]. During open coding, interview statements and passages are assigned with (partially in vivo) labels. After that, axial coding seeks to categorize open codes that relate to the same phenomenon and meaning. Finally, selective codes are identified that are able to describe and subsume all axial codes.

The coding procedure was performed in three steps, following and adapting the procedure described by Mueller and Heger [24]. First, two of the authors independently coded the data. This led to two separate schemes including open, axial, and selective codes. Second, the authors discussed and compared their schemes. For that, open and axial codes were reframed, partially renamed, and finally merged into a new categorization scheme. Next, the authors analyzed whether the identified selective codes 
withstand or new categories arise. This led to a new comprehensive scheme involving 4 selective codes and 9 axial codes. Table 1 shows a coding example. Third, each author re-coded the data according to the agreed coding scheme. Subsequently, the assignment of elaborated codes to the data was discussed once more, resolving disagreements and yielding in a final data coding.

Table 1. Coding example

\begin{tabular}{|l|l|l|}
\hline Open Code & Axial Code & $\begin{array}{l}\text { Selective } \\
\text { Code }\end{array}$ \\
\hline $\begin{array}{l}\text { "In the past [...] you } \\
\text { have looked something } \\
\text { up in books or } \\
\text { magazines, today you } \\
\text { visit the Internet. The } \\
\text { self-treatment is not } \\
\text { wrong, takes place at } \\
\text { any time [...]" }\end{array}$ & $\begin{array}{l}\text { Pelf- } \\
\text { Information }\end{array}$ & \\
(Interviewee 6) & & \\
\hline $\begin{array}{l}\text { Activation } \\
\text { with [...] the most } \\
\text { dramatic and severe } \\
\text { they could find [...] } \\
\text { and so they arrive } \\
\text { already frightened } \\
\text { because they can't deal } \\
\text { with what they read } \\
\text { and ultimately can't } \\
\text { classify it." }\end{array}$ & \\
(Interviewee 4) & & \\
\end{tabular}

\section{Findings}

Based on the seven interviews, we built four main categories to generalize relevant content regarding our research question: (1) Patient Activation, (2) Impacts on Treatment Process, (3) Patient Differentiation, and (4) Patient-Physician-Interaction. Each main category comprises subcategories, which can be considered as an accumulation of axial codes. To prevent the potential identification of interviewees, for instance by delineating interviews by means of their order, we assigned each interviewee a random number [24].

\subsection{Patient Activation}

We divided the main category Patient Activation into three subcategories: patient self-information, patient insecurity / unwanted framing and patient motivation. The first subcategory patient selfinformation contains statements about the effects of a digitally assisted confrontation of the patients with their own symptoms or process of disease from the physician's perspective. Frequently, those statements refer to a patient self-initiated internet research of symptoms before visiting the physician's practice. The majority of physicians appreciated a proactive informational process of patients: "I even recommend doing a research, but for example I mention patient organization [...]. There are even apps for young people from companies containing fantastic information [...]." (Interviewee 7). Two participants highlighted the benefits of a preceded research by the patient because of its impact on a dynamic therapeutic process: "[...] I like that, because it's always good if there is an informed patient than to explain everything from the beginning. Most of the time, patients actually know relatively well what it is about [...]" (Interviewee 5) or "[...] Actually, I appreciate it when there is a patient with advance information. This way, you are able to sort things out for them, sort things out with them together." (Interviewee 2). In contrast, the second subcategory patient insecurity / unwanted framing describes the physician's view on mostly negative effects from self-initiated internet research. These effects are related to a process of manifesting a special belief or assumption of a possible diagnosis for the patient's own symptoms or rather pathology: "Well, the middle generation, youths as well, they are reading a lot on Wikipedia and sometimes, as a result, there appear some curious things and of course this is what makes them feel even more insecure [...]." (Interviewee 6) or "Most of them got anything from Google. Most of the time, always, it's just the most dramatic and severe they could find. A small pigmentation becomes syphilis and so they arrive already frightened because they can't deal with what they read and ultimately can't classify it." (Interviewee 4). While Interviewee 4 and 6 describe tendencies of the patient to consider the most pessimistic interpretation of specific symptoms because of a lacking ability to differentiate, one physician mentioned the patient's general need to interpret their symptoms as a possible explanation: "Sometimes a lot of those stressing reactions are simply masked, people are looking for something to fit their symptoms and don't recognize where it is actually coming from." (Interviewee 3). The participants described consequences of those unwanted effects as well: "Well, in this way, you always encourage illnessawareness as well, not just health-awareness. That is not good." (Interviewee 5) or "[...] you are questioned when you don't say the same thing that is on the internet, then you aren't a good physician, I mean then, you didn't think of it as an important thing to mention [...]" (Interviewee 3). While the first two subcategories of Patient Activation refer to different 
facets of an informational process, patient motivation characterizes an actual intention to act due to collected information or technological opportunities. Physicians portrayed hypothetical and present motivational effects relating to digital technology, e.g., a shortened time period until a therapy might start or the timesaving use of online requests for prescriptions: "[...] but some might come earlier, so that they don't delay three weeks, but have a shorter way [...]" (Interviewee 3) or "[...] but also elderly patients manage to use it well or ask their relatives for help and don't make their request personally here in our practice, but the electronic way is used more and more." (Interviewee 2). Built from the subcategories, Patient Activation comprises patients' use of technologies to self-manage their own symptoms or process of disease with different outcomes on a therapeutic situation concerning physician and patient, while the physicians' perspectives include hypothetical and actual effects.

\subsection{Impacts on Treatment Process}

We divided Impacts on Treatment Process into the subcategories effort reduction/increase and technological reliability. While the hypothesis appears appropriate, that nearly any factor concerning the digitalization of primary care practices might influence the treatment process itself, some aspects of the mentioned subcategories were stated explicitly in the interviews. In our first subcategory, physicians state their believes and experiences in terms of beneficial or adverse use of digital technologies as well as perceived technological boundaries for therapy. On the one hand, high expectations of positive technological effects are mentioned: "[a faster] communication [...] with a safe connection, that would make work easier." (Interviewee 6) or "With help from telemedicine technology you could spare some time...he [the patient] doesn't have to come, I don't have to visit...that's a real advantage." (Interviewee 5). In this context, not only aspects of a time-winning communication were referred to, but also hypothetical advantages of new data infrastructures: "[...] you got access to patient's data fast, e.g., from a cloud [...]" (Interviewee 6) or "Home visits cost a lot of time. If you are able to select by necessity [...] you could save a lot of time." (Interviewee 1). On the other hand, sceptical quotes were extracted from the interviews. Participants worried about additional efforts caused by new technology: "All in all it [telemedicine] might be useful for trivialities, but the time it takes might exceed the time I spend during consultation hours." (Interviewee 6) or "If you have to differentiate it [data] yourself in the first place, I don't know if it really is time saving." (Interviewee 6) or "No, it's not helpful. It [patient's insecurity through internet research] takes time." (Interviewee 7). One physician concludes that complexity and usability of technology might be a reason for reservation: "It is [online appointment allocation] not wanted in our practice, because it's said that nobody is able to operate it, to manage it technically." (Interviewee 3). Furthermore, one physician drew parallels to a consumer-oriented selfconception of patients causing additional efforts: "[...] if every patient [...] has the right to consult his physician via monitor, you are faced with an uncontrollable flood of demands. That won't work of course." (Interviewee 5). Conclusively, physicians formulate technological reliability as an important factor concerning Impacts on Treatment Process. Differentiated from possible boundaries, a lack of security actually worries or scares the interviewees: "Strangers having access to data or misusing data, possibly having different interests than our patients." (Interviewee 1) or "I'm noticing a huge risk in not realising a decent data security." (Interviewee 5) or "Privacy, that is important [...] see what's already been hacked, we're scared of course." (Interviewee 4). Together, both mentioned subcategories explicate conditions and hypothetical effects of a medical digitalization from the physicians' point of view, illustrating technology-related expectations.

\subsection{Patient Differentiation}

During the interviews, physicians outlined specific differences between potential or actual users of healthcare-related digital technology. We merged those contents to our third main category Patient Differentiation, including the two subcategories case dependency/characteristics and stereotyping. While the first category contains statements in which physicians explained or justified a differentiated hypothesis about patient's use of technology, the second category includes heuristic and generalized statements about a large group of patients or people. As an example for the first subcategory case dependency/characteristics, one physician emphasizes individual technical skills and competencies of their patients: "That's [use of online requests for prescriptions] totally various. Most of our patients under 30, of course, but our elderly patients [...] as well [...] are getting help from their relatives [...]" (Interviewee 2). Additionally, physicians differentiated the benefit of technology use with regard to specific patient groups, e.g., known/unknown patients, chronical/non-chronical patients, and severe/non-severe diseases: "Concerning 
chronical patients it [telemedicine] might be useful [...]. As said before, in exceptional cases [...] for bedridden patients, patients with a severe disease [...]" (Interviewee 5). Besides type and seriousness of disease, the status of a personal relationship between physician and patient was seen relevant: "Anyway, I would only consider it [telemedicine] useful for patients I already know. Where I know their surroundings." (Interviewee 1). One physician expressed their idea of filtering patients that might be able to use and accept telemedicine services: "Eventually, you need someone to select those patients fitting [...]" (Interviewee 3). Another participant concludes a need for individually configurable algorithms analysing patient data: "Geriatric patient's measurements [...] it needs different thresholds [...] I should be able to determine a threshold value for an algorithm [...] a standardized configuration, that's not possible." (Interviewee 2). Unlike examples of underlining individual characteristics of patients' technological competencies, several statements of the participants generalize patient groups in terms of their age or place of residence: "In urban areas I envisage a use of online appointment allocation [...]. Here, that's not possible." (Interviewee 6) or "Our generation, surely [accept telemedicine services] but not elderly people, they struggle with those things." (Interviewee 3) or "I think it depends on their age [use of telemedicine services]" (Interviewee 3). As well as the other two main categories, Patient Differentiation shows an ambiguity between individualizing and generalizing patient characteristics.

\subsection{Patient-Physician-Interaction}

In our last main category, we explicate the physicians' statements concerning their experience in their therapeutic relationship to a patient. In contrast to Impact on Treatment Process, the category PatientPhysician-Interaction does not include general aspects but ones of direct, situated interaction between patient, physician, and technological artefacts. We divided this category into the two subcategories physician-patient collaboration and personal/bodily contact. The first subcategory concerns strategies to actively deal with a modified informational state of patients using online sources to fathom their symptoms: "Everyone has ideas about something. I've got my ideas as well and as a consequence [...] we try to bring them together [...]" (Interviewee 6) or "I'm telling my patients: You might read everything you like, write it all down, but visit me afterwards and talk with me about it." (Interviewee 7). In this context, one physician pointed out the need to accept the patient's own research to integrate it into the therapeutic process: "That [patient's worry about self-researched symptoms] is just the way it is - you have to take care of it, you have to sort things out." (Interviewee 4). Therefore, physicians state in which ways they deal with effects of modern information technology (as mentioned within the main category Patient Activation). While it might appear conceivable that the participants mention similar solutions for other technological novelties, especially telemedicine services are considered more of a limiting aspect for interaction. Physicians underline the consequent lack of personal or bodily contact constituting the second subcategory: "When complex problems occur, it is sometimes important, to have personal contact [...] that means to meet the person and see his surroundings." (Interviewee 1). Two physicians described their intuitional perception of a patient as an important factor for diagnosis and the lack of it using telemedicine systems: "You are feeling it, don't you? And that's absent in a video [...] you can't touch him or her [patient]." (Interviewee 6) or "And [...] I don't feel the patient. I can't describe it [...] you got a feeling that is appropriate most of the time." (Interviewee 6). The decrease of social interaction within a therapeutic process through a telemedicine system appeared to be another possible reason for a rejecting position: "Because personal contact is very important, especially for elderly patients or those in need of home visits being helpless [...]" (Interviewee 2). One physician summarized the perceived disadvantages: "Generally, contact between physician and patient is always important [...] the way somebody speaks, acts, walks through the door... are things a video can't show [...]" (Interviewee 5). While these statements sound resolute, more relativizing perspectives can also be reported from one interview: "[...] sometimes it is important to touch a patient. It doesn't have to be at the first visit, but it has to be possible some time during the treatment process [...]" (Interviewee 2) and "[...] because fundamental trust is necessary, you can't gain it electronically and for patient's treatment it is mandatory." (Interviewee 2). Hence, our last main category describes a physician's direct involvement with challenges arriving through aspects of medical digitalization, differentiated in already experienced solution strategies and hypothetical limits of telemedicine services.

\section{Discussion}

Noteworthy, physicians contrasted the patients' self-management in the form of self-initiated research concerning symptoms or their process of disease through (1) more positively perceived and (2) more 
negatively perceived Patient Activation. One might assume that from the physicians' perspective, the outcome of a patient's self-initiated process of information, which can be viewed as a digitally assisted empowerment, depends on his ability to select, sort, and analyze information relating to their symptoms or process of disease. While a neutrally informed patient appears to be preferable, a deep analysis of information might frame a patient so sustainably, physicians actually perceive a need to revise the patient's belief resulting in a time-costly effort and/or a negative effect on the relationship between patient and physician. To reconstruct a patient's self-initiated informational process might be an interesting approach for future work to understand motives and motivations, probably on a way to a selfmade diagnosis. Subsequent work might be able to formulate implications for a precise and self-reflected way for patients to gather health-related information affecting themselves or relatives.

By means of the second main category, Impacts on Treatment Process, the physicians' expectations related to digital technology use in practice can be described simultaneously. Concerning our interviews, the main factor to measure reduction or increase of effort might be the time spent on a specific task. Due to the fact that many physicians in rural areas have a high case ratio, a deducted hypothesis might be that the benefit of digital technology and the intention to use it depend on the actual time saved or caused by it. As a factor probably predicting a physician's intention to use health-related digital technology or as a measure of technological usability, 'time spent on a specific task' might be considered as a variable in future studies (especially as a pre-post comparison), but not without critically reflecting on an increase in efficacy for merely economic reasons.

With regard to a differentiated view on a patient's technological abilities and intentions, physicians considered the individual use of technology they already knew (e.g., online requests for prescriptions) or thought helpful (e.g., telemedicine services for well-known patients), positive in some cases. Considering a specific technological novelty not practical or useful, physicians underlined basic differences between patient groups (e.g., old vs. young). Cautiously hypothesized from quotes of our main category Patient Differentiation, physicians might sometimes justify a misuse of technology with stereotypes or generalized statements about their patients. Discussing stereotypes as well as perceived barriers of technological use and clarifying actual technical obstacles might be taken into account as an important aspect of health-related technological implementation and practice.
Statements from the main category PatientPhysician-Interaction demonstrate a physician's strategy to adopt technological aspects concerning their relationship with patients directly. To deal with partially worrying or misinterpreted information patients gather from online research, physicians formulated an understanding dialogue or process of negotiation as a possible solution. This might be considered an interesting example for an adaption of digital-technological change into the relationship between physician and patient. Besides, physicians seem to consider a personal or bodily presence of the patient an essential component of the relationship between patient and physician. Despite relativization, absence might function as a limiting factor of technological novelties and their implementation. This result can be seen as a possible impulse to increase theoretical work on phenomenological approaches to digital technology in healthcare, as it already is discussed in the medical field [4]. Similarly, specific technological requirements, such as the necessity to see the patient within their surroundings (i.e., not isolated from it) or to experience a haptic feedback, are implicitly stated in our interviews. For us, especially the seeming contradiction of telemedicine services and sophisticated primary care is considered an innovative and challenging field for future work.

\section{Conclusion \& Outlook}

Following our research question, we examined factors constituting physicians' perspectives on patient empowerment through digital technology. Findings of seven interviews with primary care physicians suggest that level and quality of informational knowledge attained through online resources affect the view on a patient. While an objective and reserved handling of information by the patient is seized as an improving factor, physicians may consider a restricted belief about a patient's own symptoms disruptive. Additionally, the interviewees valued the usability of digital health-related technologies as well as possible resulting empowerment of patients according to the effort of time needed to fulfill a task with or without its help. Furthermore, the interviewed physicians classified patients' capabilities of technology use due to their individual or general characteristics (e.g., morbidity, age). As a constituting factor, physicians described the direct effect of technology on a personal (bodily) relationship between themselves and their patients.

Our empirical study underlies some limitations. First, we did not differentiate our sample and the gathered data by areas of expertise and potential 
specializations. Whereas all of the interviewees are practicing in primary care, some of them are specialists in certain areas (e.g., diabetes). In addition, prior studies identified age influencing IT adoption and assessment [25], which we did not factor in as well. Further, all participants reside in the same area, thus neglecting potential regional differences (e.g., with regard to population size and characteristics). Considering these differences during data collection and analysis could yield new insights. Second, we have engaged a rather small sample size using a convenient approach, affecting the external validity of our findings. Addressing a wider population, for instance by deploying quantitative methods such as broad surveys, yields more generalizable insights.

Our study at hand opens up several future research opportunities. First, our findings motivate the formulation of hypotheses testing the effect identified factors (i.e., main categories) have on important dependent variables such as behavioral use intentions and actual IT adoption and use behavior. For instance, perceived patient insecurity occurring when using health technologies might negatively influence physicians' intentions to use such a system. Second, the investigation of patient-sided perspectives on the digitalization of treatments and the accompanying empowerment through digital tools can deliver novel, complementary, or even conflicting insights. The comparison of health consumers and providers, thus, represents a fruitful avenue for subsequent studies.

\section{References}

[1] Ahmad, F., P.L. Hudak, K. Bercovitz, E. Hollenberg, and W. Levinson, "Are Physicians Ready for Patients With Internet-Based Health Information?", Journal of Medical Internet Research 8(3), 2006, pp. e22.

[2] Apt, N.A., "Ageing and the changing role of the family and the community: An African perspective", International Social Security Review 55(1), 2002, pp. 39-47.

[3] Brems, C., M.E. Johnson, T.D. Warner, and L.W. Roberts, "Barriers to healthcare as reported by rural and urban interprofessional providers", Journal of Interprofessional Care 20(2), 2006, pp. 105-118.

[4] Carel, H., "Phenomenology and its application in medicine", Theoretical Medicine and Bioethics 32(1), 2011, pp. 33-46.

[5] Castro, E.M., T. Van Regenmortel, K. Vanhaecht, W. Sermeus, and A. Van Hecke, "Patient empowerment, patient participation and patient-centeredness in hospital care: A concept analysis based on a literature review", Patient Education and Counseling 99(12), 2016, pp. 19231939.
[6] Chau, P.Y.K., and P.J.-H. Hu, "Investigating healthcare professionals' decisions to accept telemedicine technology: an empirical test of competing theories", Information \& Management 39(4), 2002, pp. 297-311.

[7] Currie, M., L.J. Philip, and A. Roberts, "Attitudes towards the use and acceptance of eHealth technologies: a case study of older adults living with chronic pain and implications for rural healthcare", BMC Health Services Research 15(1), 2015, pp. 162-174.

[8] Davis, S., and H. Bartlett, "Healthy ageing in rural Australia: Issues and challenges: Rural healthy ageing", Australasian Journal on Ageing 27(2), 2008, pp. 56-60.

[9] Demiris, G., and B.K. Hensel, "Technologies for an aging society: a systematic review of 'smart home' applications", Yearbook of medical informatics 17(01), 2008, pp. 33-40.

[10] Emanuel, E.J., and L.L. Emanuel, "Four models of the physician-patient relationship", JAMA 267(16), 1992, pp. 2221-2226.

[11] Grubaugh, A.L., G.D. Cain, J.D. Elhai, S.L. Patrick, and B.C. Frueh, "Attitudes Toward Medical and Mental Health Care Delivered Via Telehealth Applications Among Rural and Urban Primary Care Patients":, The Journal of Nervous and Mental Disease 196(2), 2008, pp. 166-170.

[12] Hartley, D., "Rural Health Disparities, Population Health, and Rural Culture", American Journal of Public Health 94(10), 2004, pp. 1675-1678.

[13] Hojat, M., D.Z. Louis, K. Maxwell, F. Markham, R. Wender, and J.S. Gonnella, "Patient perceptions of physician empathy, satisfaction with physician, interpersonal trust, and compliance", International Journal of Medical Education 1, 2010, pp. 83-87.

[14] Jimison, H., P. Gorman, S. Woods, et al., "Barriers and drivers of health information technology use for the elderly, chronically ill, and underserved", Evid Rep Technol Assess (Full Rep) 175, 2008, pp. 1-1422.

[15] Johnson, M.E., C. Brems, T.D. Warner, and L.W. Roberts, "Rural-Urban Health Care Provider Disparities in Alaska and New Mexico", Administration and Policy in Mental Health and Mental Health Services Research 33(4), 2006, pp. 504-507.

[16] Karsh, B.-T., M.B. Weinger, P.A. Abbott, and R.L. Wears, "Health information technology: fallacies and sober realities", Journal of the American Medical Informatics Association 17(6), 2010, pp. 617-623.

[17] Kayser, L., A. Kushniruk, R.H. Osborne, O. Norgaard, and P. Turner, "Enhancing the Effectiveness of ConsumerFocused Health Information Technology Systems Through 
eHealth Literacy: A Framework for Understanding Users' Needs", JMIR Human Factors 2(1), 2015, pp. e9.

[18] Kvedar, J., M.J. Coye, and W. Everett, “Connected health: a review of technologies and strategies to improve patient care with telemedicine and telehealth", Health Affairs 33(2), 2014, pp. 194-199.

[19] Luxton, D.D., R.A. McCann, N.E. Bush, M.C. Mishkind, and G.M. Reger, "mHealth for mental health: Integrating smartphone technology in behavioral healthcare", Professional Psychology: Research and Practice 42(6), 2011, pp. 505-512.

[20] Martínez, A., V. Villarroel, J. Seoane, and F. Pozo, "Rural telemedicine for primary healthcare in developing countries", IEEE Technology and Society Magazine 23(2), 2004, pp. 13-22.

[21] Meher, S.K., R.S. Tyagi, and T. Chaudhry, "Awareness and attitudes to telemedicine among doctors and patients in India", Journal of Telemedicine and Telecare 15(3), 2009, pp. 139-141.

[22] Meyer, J., S. Simske, K.A. Siek, C.G. Gurrin, and H. Hermens, "Beyond quantified self: data for wellbeing", CHI'14 Extended Abstracts on Human Factors in Computing Systems, ACM Press (2014), 95-98.

[23] Misra, V., A. Bozkurt, B. Calhoun, et al., "Flexible Technologies for Self-Powered Wearable Health and Environmental Sensing", Proceedings of the IEEE 103(4), 2015, pp. 665-681.

[24] Mueller, M., and O. Heger, "Health at Any Cost? Investigating Ethical Dimensions and Potential Conflicts of an Ambulatory Therapeutic Assistance System through Value Sensitive Design", Proceedings of the 39th International Conference on Information Systems (ICIS), San Francisco, USA, 2018.

[25] Niehaves, B., and R. Plattfaut, "Internet adoption by the elderly: employing IS technology acceptance theories for understanding the age-related digital divide", European Journal of Information Systems 23(6), 2014, pp. 708-726.

[26] Osei-Frimpong, K., A. Wilson, and F. Lemke, "Patient co-creation activities in healthcare service delivery at the micro level: The influence of online access to healthcare information", Technological Forecasting and Social Change 126, 2018, pp. 14-27.

[27] Pantelopoulos, A., and N.G. Bourbakis, "A Survey on Wearable Sensor-Based Systems for Health Monitoring and Prognosis", IEEE Transactions on Systems, Man, and Cybernetics 40(1), 2010, pp. 1-12.

[28] Politzer, R.M., J. Yoon, L. Shi, R.G. Hughes, J. Regan, and M.H. Gaston, "Inequality in America: The
Contribution of Health Centers in Reducing and Eliminating Disparities in Access to Care", Medical Care Research and Review 58(2), 2001, pp. 234-248.

[29] Risk, A., and C. Petersen, "Health information on the internet: quality issues and international initiatives", Jama 287(20), 2002, pp. 2713-2715.

[30] Rosenblatt, R.A., and L.G. Hart, "Physicians and rural America", Western Journal of Medicine 173(5), 2000, pp. 348.

[31] Sittig, D.F., and H. Singh, "Legal, Ethical, and Financial Dilemmas in Electronic Health Record Adoption and Use", PEDIATRICS 127(4), 2011, pp. e1042-e1047.

[32] Strauss, A.L., and J.M. Corbin, Basics of qualitative research: techniques and procedures for developing grounded theory, Sage Publications, Thousand Oaks, 1998.

[33] Thommasen, H.V., C.M. Lavanchy, C.I. Connelly, J. Berkowitz, and S. Grzybowski, "Mental health, job satisfaction, and intention to relocate. Opinions of physicians in rural British Columbia", Canadian Family Physician 47(4), 2001, pp. 737-744.

[34] Van Woerkum, C.M., "The Internet and primary care physicians: coping with different expectations", The American Journal of Clinical Nutrition 77(4), 2003, pp. 1016-1018.

[35] Wiederhold, B.K., "mHealth Sensors Can Promote Behavior Change and Reduce Healthcare Costs", CyberPsychology, Behavior \& Social Networking 18(10), 2015, pp. 559-560.

[36] Wilson, N., I. Couper, E. De Vries, S. Reid, T. Fish, and B. Marais, "Inequitable distribution of healthcare professionals to rural and remote areas", Rural Remote Health 9(1060), 2009.

[37] Yang, J., "Potential urban-to-rural physician migration: The limited role of financial incentives", Canadian Journal of Rural Medicine 8(2), 2003, pp. 101106.

[38] Zahnd, W.E., S.L. Scaife, and M.L. Francis, "Health literacy skills in rural and urban populations", American journal of health behavior 33(5), 2009, pp. 550-557.

[39] Zhong, H., "The impact of population aging on income inequality in developing countries: Evidence from rural China", China Economic Review 22(1), 2011, pp. 98-107. 